\title{
Quantification du thromboxane B2 par CLHP-ESI-SM • Application à des microsomes de levure exprimant la thromboxane synthase humaine
}

\section{LC-ESI-MS quantification of thromboxane B2 in yeast microsomes expressing human thromboxane synthase}

Dany CHEVALIER ${ }^{(1,2) *}$, Florian KLINZIG ${ }^{(3)}$, Luc HUMBERT $^{(3)}$, Christelle CAUFFIEZ $^{(2)}$, Jean-Marc LO-GUIDICE ${ }^{(2)}$, Delphine ALLORGE ${ }^{(2)}$, Bernard CARTIGNY(1), Michel IMBENOTTE ${ }^{(1)}$, Franck BROLY ${ }^{(2,3)}$, Michel LHERMITTE ${ }^{(1,2,3)}$

(1) Laboratoire de Toxicologie, Faculté des Sciences Pharmaceutiques et Biologiques, 3, rue du Professeur Laguesse - 59006 LILLE Cedex

(2) Équipe d'Accueil 2679, Variabilité génétique des défenses de la muqueuse respiratoire face à son environnement, Faculté de Médecine, Pôle Recherche, place de Verdun - 59045 LILLE Cedex

(3) Laboratoire de Biochimie et de Biologie Moléculaire, Hôpital Calmette, CHRU de Lille, Boulevard du Professeur J. Leclercq - 59045 LILLE Cedex

*Auteur à qui adresser la correspondance : Dr Dany CHEVALIER, Laboratoire de Toxicologie, Faculté des Sciences Pharmaceutiques et Biologiques, 3, rue du Professeur Laguesse - 59006 LILLE Cedex Tél : +33 320626820 - Fax : +33 320626884 - E-mail : dany.chevalier@caramail.com 


\section{$\boldsymbol{R E} \boldsymbol{E} U M \boldsymbol{E}$}

Le but de cette étude était de mettre au point une méthode rapide, sensible et spécifique de quantification du thromboxane B2 (TXB2) par chromatographie liquide haute performance couplée à une interface d'ionisation électrospray et à une détection par spectrométrie de masse (CLHP-ESI$S M)$, à partir de microsomes de levure exprimant la thromboxane synthase (CYP5AI) humaine. Ce métabolite est le produit d'hydratation stable du thromboxane A2 (TXA2) produit par le CYPSA1 à partir de son substrat, la prostaglandine H2 (PGH2). La quantification du TXB2 a été réalisée à l'aide d'un standard interne deutéré, le 6-céto-PGFI $\alpha$ d4, ajouté avant une extraction par le diéthyléther. La séparation chromatographique s'effectue en phase inverse sur colonne XTerra MS C18. L'élution est réalisée en mode isocratique $(60 \%$ acétonitrile contenant $0,05 \%$ d'acide formique - $40 \%$ tampon formiate d'ammonium $5 \mathrm{mM}, \mathrm{pH} 3,0$ ). Le mode d'ionisation électrospray négatif a été retenu pour une meilleure détection du TXB2 et du 6-céto-PGFI $\alpha$-d4. Cette technique appliquée avec succès aux microsomes exprimant le CYP5A1 sauvage montre une production croissante et linéaire de TXB2 pour la gamme de concentrations de PGH2 utilisée $(0-120 \mu M)$. Cette méthode pourra être appliquée à l'analyse de l'activité enzymatique de nouveaux variants de la thromboxane synthase, récemment mis en évidence au laboratoire, et exprimés dans des microsomes de levure.

\section{MOTS-CLÉS}

Thromboxane B2, 6-céto-PGFI $\alpha$-d4, CLHP-ESI-SM, thromboxane synthase (CYPSAI) humaine, microsome.

\section{Introduction}

La thromboxane synthase (CYP5A1, EC 5.3.99.5) est une isoenzyme appartenant à la superfamille des cytochromes P450 (CYP450) (1). Ces isoenzymes sont impliquées dans la détoxication de molécules exogènes, comme les médicaments ou les polluants environnementaux $(2,3)$, mais également dans le métabolisme de composés endogènes, comme les stéroïdes, les acides biliaires, les acides gras, les leucotriènes, les prostaglandines et les thromboxanes (4). Le CYP5A1 est le seul représentant de la famille 5 des CYP450. Il intervient dans la cascade d'activation de l'acide arachidonique par la voie de la cyclooxygénase et catalyse l'isomérisation de la prostaglandine $\mathrm{H} 2$ ( $\mathrm{PGH} 2)$ en thromboxane A2 (TXA2). Ce.dernier se décompose spontanément (demi-vie $30 \mathrm{sec}$ ), en métabolite inactif stable, le thromboxane B2 (TXB2) (Figure 1) (5). Le TXA2 joue un rôle important dans de nombreux processus physiopathologiques. C'est un inducteur de l'agrégation plaquettaire, de l'inflammation, de la vasoconstriction, de la bronchoconstriction et il participe à la régulation de l'hémostase $(6,7)$. Produit en excès, il contribue au développement de pathologies sévères comme l'athérosclérose, l'infarctus cérébral,

\section{SUMMARY}

An LC-ESI-MS method for specific, sensitive and rapid quantitative analysis of thromboxane B2 (TXB2) in yeast microsomes expressing human thromboxane synthase (CYP5AI) was developed. TXB2 is a stable, biologically inert metabolite formed from the non-enzymatic hydrolysis of TXA2, which is produced by thromboxane synthase (CYP5A1) from prostaglandin H2 (PGH2). TXB2 was quantified after addition of 6-céto-PGF1 $\alpha$-d4 as an internal standard, by liquid phase extraction using diethylether and reverse phase LC-ESI-MS. HPLC/MS analysis was performed with a column C18 XTerra using a mobile phase $(60 \%$ acetonitrile with $0.05 \%$ formic acid - $40 \%$ ammonium formiate $5 \mathrm{mM}, \mathrm{pH}$ 3.0). The LC system used ion spray interface with MS system in negative ion detection and selected ion recording mode. The method was successfully applied to yeast microsomes expressing human CYPSAl incubated with $P G H 2$, and $T X B 2$ production appears to be linear for $P G H 2$ concentrations ranging from 0 to $120 \mu \mathrm{M}$. This method could be used for the kinetic analysis of new CYP5Al variants expressed in yeast microsomes, recently identified in our laboratory.

\section{KEY-WORDS}

Thromboxane B2, 6-keto-PGFI $\alpha$-d4, LC-ESI-MS, human thromboxane synthase (CYP5A1), microsome.

l'hypertension artérielle pulmonaire et l'asthme bronchique $(8,9)$. Cliniquement, un déficit d'activité du CYP5A1 a été rapporté chez des patients présentant des troubles hémorragiques intestinaux (10-12). Cependant, l'origine moléculaire de ce déficit n'a pas été mise en évidence. Récemment, de nouveaux variants génétiques de la thromboxane synthase ont été identifiés par notre équipe (13). Dans le but d'analyser leurs conséquences fonctionnelles sur l'activité thromboxane synthase, nous avons utilisé un système cellulaire hétérologue d'expression, la levure Saccharomyces cerevisiae.

Différentes techniques sont proposées pour l'analyse du TXB2, produit d'hydratation stable du TXA2, comme la radioimmunologie (RIA) (14), la chromatographie liquide haute performance en détection UV (CLHP-UV) $(15,16)$ et la chromatographie phase gaz couplée à la spectrométrie de masse (CPG-SM) (17, 18) mais elles présentent certaines limites. Celles de la RIA sont dues à une faible spécificité et à un manque de sensibilité, ce qui se retrouve pour la CLHP-UV. Quant à la CPG-SM, de nombreuses étapes de dérivation sont nécessaires et relativement longues à mettre en oeuvre. Il apparaît donc important de développer une méthode rapide, sensible et spécifique de quantifi- 


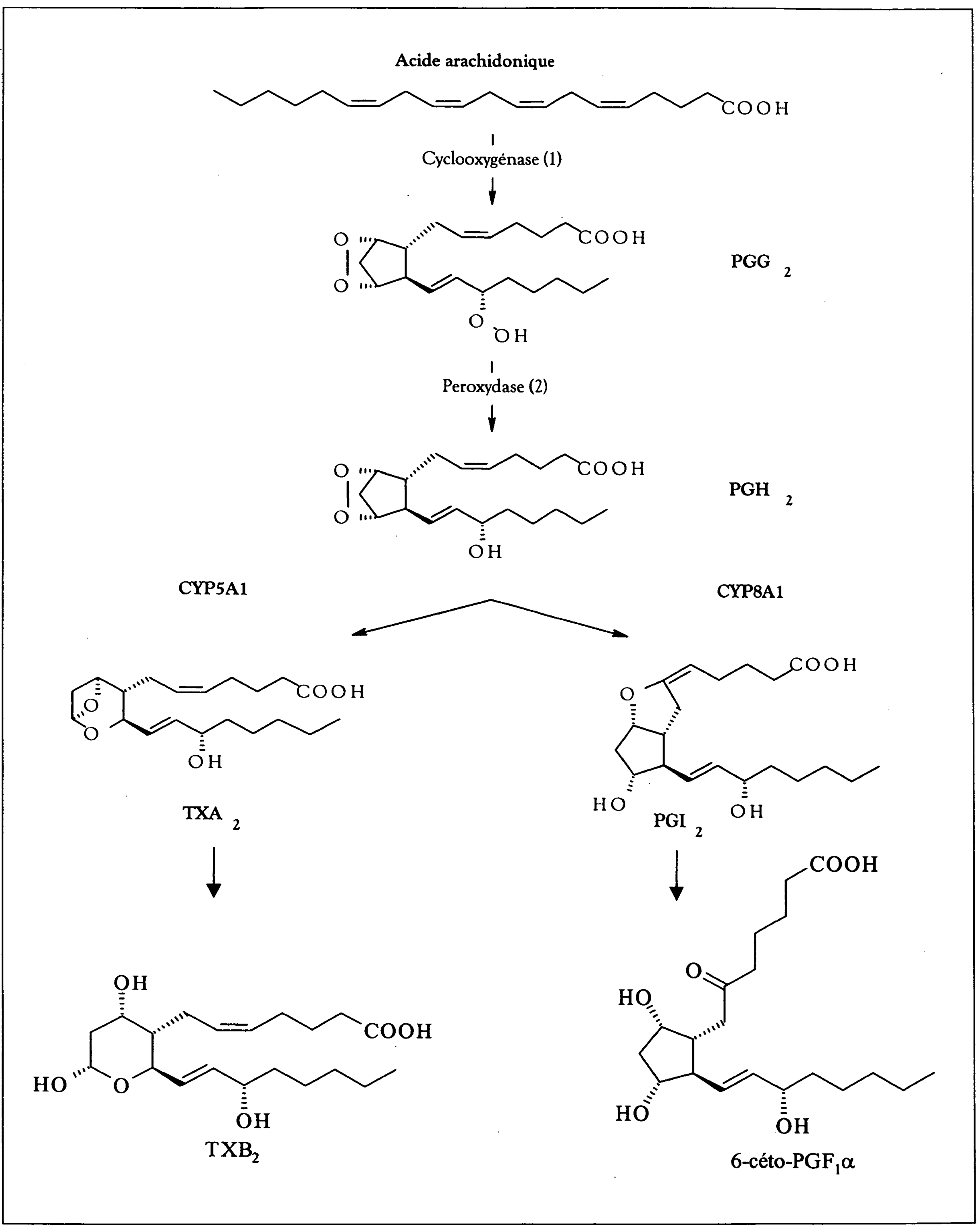

Figure I : Métabolisme de l'acide arachidonique par la voie de la cyclooxygénase. Le site cyclooxygénase de la prostaglandine endoperoxyde G/H synthase (PGHS ou COX) catalyse la réaction en (1), le site peroxydase de la même enzyme catalyse la réaction en (2). L'endoperoxyde PGH2, eicosanoïde pivot, permet la synthèse des prostanoïdes TXA2 et PGI2. Le TXA2 et la PGI2 se décomposent spontanément en milieu aqueux respectivementy en TXB2 et 6-céto PGF1 $\alpha$, produits d'hydrolyse inactifs. 
cation du TXB2. Dans cette étude, nous décrivons une méthode par chromatographie liquide haute performance couplée à une interface d'ionisation électrospray et à une détection par spectrométrie de masse (CLHPESI-SM). Cette technique pourra ensuite être appliquée aux microsomes de levure exprimant les nouveaux variants du CYP5A1.

\section{Matériel et méthodes Réactifs}

Le substrat (PGH2) de la thromboxane synthase provient de chez Biomol (Plymouth Meeting, USA). Le métabolite (TXB2) et le standard interne (6-céto-prostaglandine $\mathrm{F} 1 \alpha-\mathrm{d} 4$, contenant quatre atomes de deutérium en positions $3,3^{\prime}, 4$ et 4') proviennent de chez Cayman Chemicals (Ann Arbor, USA). L'acétonitrile Chromasolv (Carlo Erba, Italie), utilisé pour la phase mobile, est de qualité CLHP. L'acide formique, le formiate d'ammonium, ainsi que le diéthyléther, proviennent de chez Sigma (St Louis, USA). Les autres réactifs utilisés sont de qualité analytique.

\section{Incubation}

Le protocole d'incubation des microsomes de levure exprimant le CYP5A1 humain a été adapté à partir des méthodes développées par Hatae et coll. (19) et Wang et coll. (20). Ainsi 100 picomoles de CYP5A1 sont mélangées dans $250 \mu \mathrm{l}$ de tampon Tris- $\mathrm{HCl} 60 \mathrm{mM}, \mathrm{pH}$ 7,4 avec le substrat $\mathrm{PGH} 2$ et incubés à $37^{\circ} \mathrm{C}$ pendant une minute. La gamme de concentrations en PGH2 s'étend de 0 à $120 \mu \mathrm{M}$. La réaction est arrêtée par l'ajout de $25 \mu 1$ d' $\mathrm{HCl} 2 \mathrm{~N}$. Après mélange au vortex et centrifugation 10 min à $3500 \mathrm{~g}$ pour précipiter les protéines, le surnageant est ajusté à $\mathrm{pH} 4,5$ avec une solution de tampon Tris 1,5 M.

\section{Extraction et quantification}

Après ajout du standard interne 6-céto-PGF1 $\alpha$-d4, pour une concentration finale de $10 \mu \mathrm{M}$ et homogénéisation du milieu, le surnageant est extrait par $3 \mathrm{ml}$ de diéthyléther. L'extrait est évaporé à sec $\left(50-70^{\circ} \mathrm{C}\right)$ sous courant d'azote et repris par $100 \mu \mathrm{l}$ de phase mobile (60\% acétonitrile contenant $0,05 \%$ d'acide formique $40 \%$ tampon formiate d'ammonium $5 \mathrm{mM}, \mathrm{pH} \mathrm{3,0)}$, puis injecté dans le système chromatographique.

\section{Instruments et conditions}

Le système CLHP-ESI-SM est composé d'un chromatographe Waters 2690 couplé à un spectromètre de masse Waters micromass ZQ équipé d'une interface électrospray et piloté par le logiciel Masslynx v 3.5. La séparation chromatographique est réalisée sur une colonne XTerra MS C18 3,5 $\mu \mathrm{m}, 150$ x 2,1 mm
(Waters), munie d'une précolonne de $10 \mathrm{~mm}$ possédant les mêmes caractéristiques et maintenues à une température de $30^{\circ} \mathrm{C}$. Après injection de $10 \mu \mathrm{l}$ de l'échantillon, l'élution est réalisée à un débit de $0,2 \mathrm{ml} / \mathrm{min}$ en mode isocratique (60\% acétonitrile contenant $0,05 \%$ d'acide formique - $40 \%$ tampon formiate d'ammonium $5 \mathrm{mM}, \mathrm{pH} 3,0$ ).

La détection du TXB2 et du 6-céto-PGF1 $\alpha$-d4 est réalisée en mode d'ionisation électrospray négatif (ES-) avec une tension d'ionisation de $3 \mathrm{kV}$ et une tension de cône de $-30 \mathrm{~V}$, associée à un enregistrement en mode fragmentométrique ou Selected Ion Recording (SIR) $(\mathrm{m} / \mathrm{z}$ 369,3 et 373,3 respectivement). L'identification et la quantification des produits sont réalisées en déterminant le temps de rétention du pic chromatographique, l'abondance relative de l'ion majoritaire de quantification et la présence des ions de confirmation.

\section{Résultats et discussion}

L'identification du TXB2 et du standard interne a été obtenue après séparation par CLHP en phase inverse après optimisation des paramètres analytiques. Une première détection, en mode «full scan», entre 100 et $650 \mathrm{~m} / z$, réalisée suivant les deux modes ES- et ES+, a permis de retenir le mode d'ionisation ES-. En effet, ce mode d'ionisation négatif a entraîné une moindre fragmentation de l'ion moléculaire.

Les caractéristiques analytiques de ces deux composés se retrouvent aussi bien à partir de solutions standards que de microsomes. La Figure 2 présente les données obtenues à partir de microsomes recombinants. Dans le chromatogramme illustrant le courant ionique total (TIC), 3 pics distincts sont observés à des temps de rétention de 3,29 $\mathrm{min}, 4,33 \mathrm{~min}$ et 5,94 min (Figure 2A). Le premier pic correspond au 6-céto-PGF1 $\alpha$-d4 (standard interne) (Figure 2B). Les spectres de masse des pics observés à 4,33 min et 5,94 min sont identiques (Figure 2D) et peuvent être attribués au TXB2 (Figure 2C). En effet ce composé donne lieu à deux formes chimiques en équilibre coexistant dans les conditions chromatographiques retenues.

En préalable à la quantification du TXB2 produit par des microsomes de levure dotés de potentiel enzymatique, une gamme d'étalonnage a été réalisée sur des microsomes n'exprimant pas le CYP5A1. Ces échantillons ont été supplémentés par des concentrations croissantes de TXB2, de 0 à $1000 \mathrm{ng} / \mathrm{ml}$. Le critère de quantification retenu est le rapport des aires des pics du TXB2 au pic du standard interne. Ceci a permis de s'assurer de la linéarité de l'étalonnage $(\mathrm{r}=0,998)$ et de préciser les. limites de détection $(2 \mathrm{ng} / \mathrm{ml})$ et de quantification $(5 \mathrm{ng} / \mathrm{ml})$ du TXB2. L'analyse dans les conditions 
décrites n'a donné lieu à aucune interférence. Le rendement de l'extraction par le diéthyléther est de $81 \%$.

Cette technique de quantification du TXB2 a ensuite été appliquée à des microsomes de levure exprimant la forme sauvage du CYP5A1. La Figure 3 montre que le rapport des aires des pics du TXB2 et du pic de 6-cétoPGF1 $\alpha$-d4 augmente de façon linéaire pour la gamme de concentrations de PGH2 utilisée $(0-120 \mu \mathrm{M})$.

Enfin, la méthode présentée pourrait être aisément transposée, avec le même standard interne, à la mesure du 6-céto-PGF1 $\alpha$ non marqué, produit par la prostacycline synthase (CYP8A1) à partir du substrat commun le PGH2 (Figure 1). Cette technique de dosage du 6céto-PGF1 $\alpha$ pourrait être appliquée à l'analyse de la forme sauvage et de variants du CYP8A1 exprimés dans des microsomes de levure (21).

\section{Conclusion}

La technique CLHP-ESI-SM apparaît comme une méthode de choix pour le dosage du TXB2. Le mode opératoire présenté, validé et appliqué avec succès à des microsomes recombinants exprimant le CYP5A1 sauvage, est plus rapide, plus spécifique et présente de meilleures limites de détection que d'autres méthodes décrites : RIA, CLHP-UV ou CPG-SM, précédemment utilisées pour le dosage du TXB2. La technique de quantification mise au point sera appliquée à la mesure de l'activité thromboxane synthase de microsomes de levure exprimant des variants du CYP5A1.

\section{Remerciements}

Cette étude a été financée par le Centre Hospitalier Régional et Universitaire de Lille, France et la Génopole de Lille, Région Nord-Pas de Calais (FEDER), France.

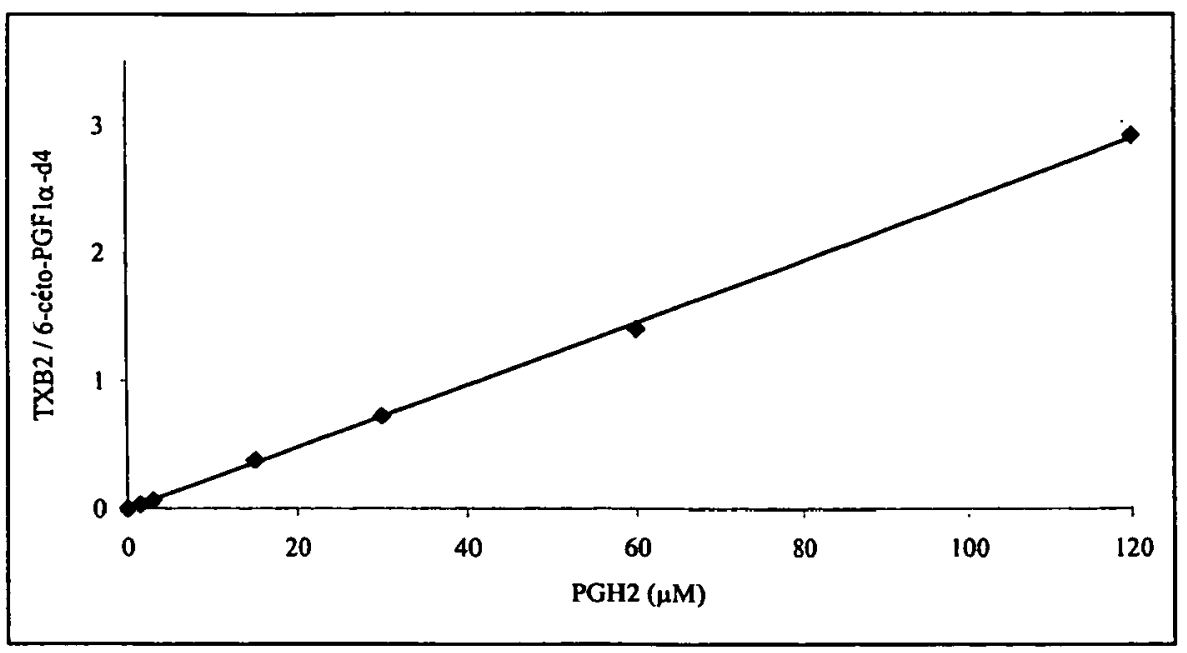

Figure 3 : Mesure par CLHP-ESI-SM du TXB2 produit par les microsomes exprimant le CYP5AI sauvage (rapport des aires des pics TXB2 / 6-céto-PGFl $\alpha$-d4) en fonction de la concentration en substrat PGH2 ( $\mu M)$ dans l'incubat.

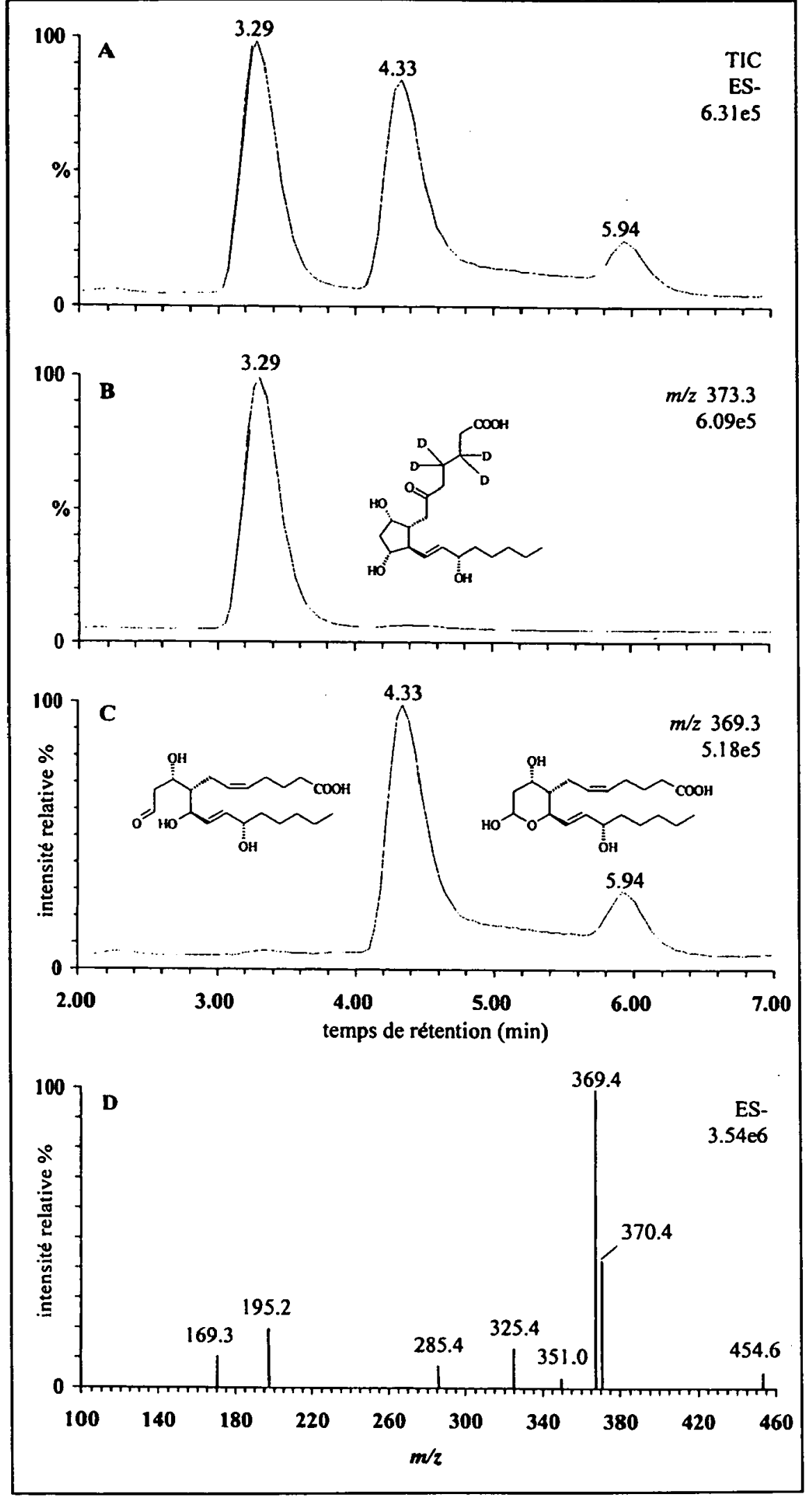

Figure 2 : Caractéristiques analytiques CLHP-ESI-SM du 6céto-PGFI $\alpha-d 4$ et du TXB2 obtenus après incubation de microsomes de levure recombinants exprimant le CYP5A1 humain avec de la PGH2. Les chromatogrammes sont les suivants : (A) courant ionique total $(B)$ ion extrait 6-céto$P G F 1 \alpha-d 4$ (standard interne) et $(C)$ ion extrait TXB2. Le double pic TXB2 est dû aux deux formes en équilibre. Les spectres SM correspond aux deux pics du TXB2 (D) sont identiques. 


\section{Références}

1. Nelson D.R., Koymans L., Kamataki T., Stegeman J.J., Feyereisen R., Waxman D.J., Waterman M.R., Gotoh O., Coon M.J., Estabrook R.W., Gunsalus I.C., Nebert D.W. P450 superfamily : update on new sequences, gene mapping, accession numbers and nomenclature. Pharmacogenetics $1996 ; 6: 1-42$.

2. Wrighton S.A., Stevens J.C. The human hepatic cytochrome P450 involved in drug metabolism. Crit. Rev. Toxicol. $1992 ; 22: 1-21$.

3. Beaune P. Human cytochromes P450. Applications in pharmacology. Therapie 1993 ; 48 : 521-6.

4. Waxman D.J., Lapenson D.P., Aoyama T., Gelboin H.V., Gonzalez F.J., Korzekwa K. Steroid hormone hydroxylase specificities of eleven cDNA-expressed human cytochrome P450s. Arch. Biochem. Biophys. $1991 ; 290$ : 160-6.

5. Needleman P., Moncada S., Bunting S., Vane J.R., Hamberg M., Samuelsson B. Identification of an enzyme in platelet microsomes which generates thromboxane A2 from prostaglandin endoperoxides. Nature $1976 ; 261$ : 558-60.

6. Whittle B.J., Moncada S. Pharmacological interactions between prostacyclin and thromboxanes. Br. Med. Bull. $1983 ; 39: 232-8$.

7. Farrukh I.S., Michael J.R., Summer W.R., Adkinson N.F., Gurtner G.H. Thromboxane-induced pulmonary vasoconstriction: involvement of calcium. J. Appl. Physiol. 1985 ; 58 : 34-44.

8. Needleman P., Turk J., Jakschik B.A., Morrison A.R., Lefkowith J.B. Arachidonic acid metabolism. Annu. Rev. Biochem. 1986 ; 55 : 69-102.

9. Ogletree M.L. Overview of physiological and pathophysiological effects of thromboxane A2. Fed. Proc. 1987 ; $46: 133-8$.

10. Weiss H.J., Lages B.A. Possible congenital defect in platelet thromboxane synthetase. Lancet $1977 ; 2: 760-1$.

11. Mestel F., Oetliker O., Beck E., Felix R., Imbach P., Wagner H.P. Severe bleeding associated with defective thromboxane synthetase. Lancet $1980 ; 1: 157$.

12. Defreyn G., Machin S.J., Carreras L.O., Dauden M.V., Chamone D.A., Vermylen J. Familial bleeding tendency with partial platelet thromboxane synthetase deficiency : reorientation of cyclic endoperoxide metabolism. Br. J. Haematol. $1981 ; 49: 29-41$.
13. Chevalier D., Lo-Guidice J.M., Sergent E., Allorge D., Debuysere H., Ferrari N., Libersa C., Lhermitte M., Broly $F$. Identification of genetic variants in the human thromboxane synthase gene (CYP5A1). Mutat. Res. $2001 ; 432: 61-7$.

14. Walenga R.W., Boone S., Stuart M.J. Re-examination of the assay for plasma prostanoids by solid-phase extraction, and radioimmunoassay. Prostaglandins Leukot. Med. $1985 ; 20: 151-67$.

15. Kiyomiya K., Yamaki K., Nimura N., Kinoshita T., Ohishi S. Phorbol myristate acetate-stimulated release of cyclooxygenase products in rat pleural cells : derivatization of prostaglandins with 9-anthryldiazomethane for fluorometric determination by high performance liquid chromatography. Prostaglandins 1986 ; 31 : 71-82.

16. Kurosawa M., Igarashi Y., Kobayashi H., Kobayashi S., Abe S. Procedures for plasma prostaglandins assay from bronchial asthmatic patients using 9-anthryldiazomethane-HPLC method. Ann. Allergy. 1990 ; 64 : 464-70.

17. Fitzpatrick F., Gorman R., Green K., Kelly R., Schneider W. Development of a gas chromatographic-mass spectrometric method using a stable isotope internal standard for quantitation of thromboxane B2. Thromb. Res. 1984 ; $35: 121-31$.

18. Schweer H., Kammer J., Seyberth H.W. Simultaneous determination of prostanoids in plasma by gas chromatography-negative-ion chemical-ionization mass spectrometry. J. Chromatogr. 1985 ; 338 : 273-80.

19. Hatae T., Hara S., Yokoyama C., Yabuki T., Inoue H., Ullrich V., Tanabe T. Site-directed mutagenesis of human prostacyclin synthase: Alteration of Cys441 of the Cyspocket, and Glu347 and Arg350 of the EXXR motif. FEBS Lett. 1996 ; 389 : 268-72.

20. Wang L.H., Matijevic-Aleksic N., Hsu P.Y., Ruan K.H., Wu K.K., Kulmacz R.J. Identification of thromboxane A2 synthase active site residues by molecular modelingguided site-directed mutagenesis. J. Biol. Chem. 1996 ; $271: 19970-5$.

21. Chevalier D., Cauffiez C., Bernard C., Lo-Guidice J.M., Allorge D., Fazio F., Ferrari N., Libersa C., Lhermitte M., D'Halluin J.C., Broly F. Characterization of new mutations in the coding sequence and 5 '-untranslated region of the human prostacyclin synthase gene (CYP8A1). Hum. Genet. 2001 ; 108 : 148-55. 